\title{
La productividad por cumplimiento de objetivos en la jefatura Central de Tráfico: hacia un nuevo modelo de gestión pública
}

Juan Antonio González Pastor*

\section{Introducción}

La jefatura Central de Tráfico - junto al resto de estrategias que componen su política de Recursos H umanos- aborda la evaluación del rendimiento en el marco de la denominada iN ueva Gestión Pública» dentro de un proceso de mejora continua en la prestación de servicios, partiendo a tal fin del análisis del cumplimiento de objetivos, la evaluación de los resultados obtenidos y la satiffacción de sus clientes internos (empleados públicos) y externos (ciudadanos).

La utilización de tecnologías de la información resulta una herra mienta imprescindible para la reducción de las cargas de trabajo, la ponderación objetiva de los trámites, la asignación de objetivos identificablesy la incentivación en función del cumplimiento delosmismos, permitiendo la implantación desistemas de userribución variables.

Se apuesta por la racionalización y la objetivación para un mejor aprovechamiento de los recursos, utilizando para ello las estrategias y mejores prácticas necesarias que permitan la optimización tanto de la calidad en el servicio al ciudadano como de las condiciones de trabajo de los empleados públicos que en él prestan sus servicios. Para ello, se confía en la adopción de sistemas incluidos dentro de la denominada «gestión de calidad» como una opción para avanzar en la mejora de la gestión públicay satiffacer las demandas ciudadanas.

Un ejemplo práctico de materialización de esta política de calidad en la Administración General del Estado es la productividad por cumplimiento de objetivos del colectivo del área administrativa y de atención al público (en adelante, CAAP) y del colectivo del área de pruebas de aptitud para la obtención de autorizaciones administrativas para conducir vehículos (en adelante, CEX) en el O rganismo Autónomo Jefatura Central de Tráfico. El objetivo perseguido es la incentivación del rendimiento a través del cumplimiento de objetivos de estas dos áreas, consideradas estratégicas en nuestra organización. Dicho programa de incentivación se desarrolla en un contexto de plantillas dimensionadas de acuerdo con el sistema de cálculo de cargas de trabajo correspondientes a cada Unidad, y en los términos que recoge el denominado «Plan Integral de Recursos H umanos», negociado con la representación sindical y firmado por cinco de los seis sindicatos acreditados en el 0 rganismo a nivel nacional.

Interesa resaltar que son puestos CAAP todos los de la organización dedicados a latramitación administrativa y atención al público, desde el Jefe Provincial o Local hasta el operador de información de nivel de complemento de destino 14.

\section{¿Por qué la evaluación del rendimiento?}

Existían varios precedentes normativos que animaban a adoptar la evaluación del rendimiento como estrategia en la política de Recursos H umanos del O rganismo Autónomo Jefatura Central de Tráfico. 


\section{El Acuerdo de Consejo de Ministros de 17 de julio de 1998}

El Consejo de Ministros, en su sesión del 17 de julio de 1998, adoptó un Acuerdo dirigido a promover la implantación de sistemas de evaluación del rendimiento de unidades administrativas en la Administración General del Estado.

Ya la Ley 6/1997, de 14 de abril, de O rganización y Funcionamiento de la Administración General del Estado (LOFAGE), supuso una reafirmación notable respecto del funcionamiento de las organizaciones administrativas estatales hacia determinados principios, entre los que se señala singularmente el de «programación y desarrollo de objetivos y control de la gestión y los resultadoss. Este enfoque de la organización y la gestión pública, basado también en la eficiencia, la calidad y la responsabilidad, se dice, debe ser la forma ordinaria de prestación de los servicios públicos, convirtiéndose ya esas prácticas directivas, de acuerdo con la LO FAGE, en un auténtico mandato legal de forma general para la Administración estatal.

M uchas serían las referencias que podrían transcribirse lite ralmente del contenido del Acuerdo del Consejo de M inistros, pero y por su extensión, a ellas nos remitimos. En cualquier caso, parece clara la apuesta del Gobierno por fomentar la autoevaluación como sistema de control interno de cada centro directivo, perfeccionar la función directiva, propiciar las prácticas de gestion orientada a los resultados e incorporar progresivamente la dirección por objetivos, estimulando la mayor eficiencia en la utilización de los recursos públicos.

\section{La norma UNE-EN ISO 9004}

La norma UNE-EN ISO 9004 de diciembre de 2000, sobre Sistemas de gestión de la calidad y D irectrices para la mejora del desempeño, es la versión oficial, en español, de la N orma Europea EN ISO 9004, de diciembre 2000, que a su vez adopta íntegramente la N orma Internacional ISO 9004:2000, y ha sido elaborada por el comitétécnico AEN /CTN 66 G estión de la Calidad, cuya Secretaría desempeña AEN OR.

Según dicha N orma, el propósito de una organización debe ser: a) identificar y satisfacer las necesidades y expectativas de sus clientes y otras partes interesadas (empleados, proveedores, propietarios, sociedad) para lograr ventaja competitiva y para hacerlo de una manera eficaz y eficiente; b) obtener, mantener, me jorar el desempeño global de una organización y sus capacidades. La N orma recuerda que la alta dirección: a) debería definir también métodos para medir el desempeño de la organización con el fin de determinar si se han al canzado los objetivos planificados; b) debería mejorar tanto la eficacia como la eficiencia de la organización. Como ayuda en el logro de sus objetivos de mejora del desempeño, la organización debería promover la participación y el desarrollo de su personal... estableciendo objetivos individuales y de equipo, gestionando el desempeño de los procesos y evaluando los resultados.

\section{El Anteproyecto de Ley Reguladora del Estatuto Básico de la Función Pública}

«Existe en la actualidad una coincidencia general en señalar que no existe hoy una gestión plenamente eficaz de los recursos humanos en las Administraciones Públicas, lo que supone un grave obstáculo en los effuerzos de modernización del aparato burocrático con el fin de convertirlo en un eficiente prestador de servicios a los ciudadanos. Esta deficiente utilización de los re cursos humanos puede ser corregida en buena medida con las medidas contenidas en el Estatuto Básco, tendente a dotar de mayor agil idad y flexibilidad la getión de personal, a motivar y estimular a los funcionarios a un cumplimiento más eficiente de sus tareas y a una mayor asunción de responsabilidades en el marco de unas Administraciones modernas quedeben legitimarseante los ciudadanos por una utilización racional y eficientede los mediosque los propios ciudadanos ponen, en último extremo, a su disposición».

Este párrafo de la Exposición de M otivos refleja claramente la apuesta que hace el Gobierno de incentivar a los empleados públicos para conseguir un más eficiente cumplimiento de sus tareas, y de asumir cada vez más responsabilidades; y alude asimismo a la «Administración moderna», caracterizada por demostrar ante los ciudadanos que su gestión es racional, eficaz y eficiente en cuanto a los medios que los ciudadanos le brindan.

La carrera diseñada en el Estatuto se desliga de la ocupación de los puestos de trabajo y se basa en la antigüedad, la trayectoria profesional, la calidad de los trabajos desarrollados, los conocimientos y la actuación profesional aplicándose a tal efecto un sistema de evaluación que supone otra importante novedad.

\section{¿Por qué en la Jefatura Central de tráfico?}

Ante las tres premisas indicadas (Acuerdo de Consejo de $\mathrm{Mi}$ nistros, Norma U N E-EN ISO 9004 y borrador del Anteproyecto de Estatuto de la Función Pública), la Jefatura Central de Tráfico no quería permanecer impasible. D e hecho, tradicionalmente había venido manifestando inquietud, talante abierto y actitud in- 
novadora en lo que respecta a los sistemas retributivos. Prueba de ello es que, antes de la publicación del Acuerdo, la Jefatura Central deTráfico había aceptado la invitación realizada por el M inisterio para las Administraciones Públicas para colaborar, como organismo piloto, para la implantación del sistema de evaluación del rendimiento (SISTEVA) elaborado por la Inspección General de Servicios de la Administración Pública, con dos fases: análisis de cuatro Jefaturas Provinciales de Tráfico (Barcelona, Sevilla, Cantabria y Toledo) y elaboración de un informe/modelo sobre el método de evaluación del rendimiento.

N uestro 0 rganismo disfrutaba por aquél entonces, y lo sigue haciendo, de una excelente situación de partida para la implantación de sistemas de evaluación del rendimiento, por cuanto que, aunque no se trata de una auténtica dirección por objetivos, ya se cuenta con una suerte de «gestión por objetivos» a nivel central, a través de lo que se denomina «Plan de Actuaciones», que se suele formalizar y traducir, en gran medida, en objetivos presupuestarios. Los J efes de cada área y actividad, a nivel central con la supervisión de los Subdirectores Generales, se comprometen a realizar anualmente una serie de objetivos (que se dividen en acciones, actividades y tareas, en función de su complejidad, duración, dedicación, trascendencia, etc...), con plazos de cumplimiento y ejecución de presupuesto, sobre la que, a su vez, se realiza el seguimiento mensual o trimestral, al nivel de la propia Dirección General. A nivel periférico, los Jefes Provinciales de Tráfico se comprometen a desarrollar en sus unidades diversas actuaciones, que son seguidas por los J efes Provinciales coordinadores, dando cuenta de ello en las reuniones trimestrales del Consejo de Dirección.

Faltaba, por tanto, la implantación experimental de un sistema de evaluación del rendimiento, objetivo asimismo incluido ya en el Plan de Actuaciones para 1999. El que la Dirección Ge neral de Tráfico incluyera en las actuaciones para 1998 y 1999 acciones relacionadas con la evaluación del rendimiento da idea de hasta qué punto se apuesta por la implantación de este tipo de sistemas e incluso - dada la infraestructura existente para medir un muy alto porcentaje de la actividad gestora, a través de numerosos indicadores y sistemas de información - por la implantación de la dirección por objetivos y por fin la evaluación del desempeño individual de cada empleado público en la producción del resultado.

La introducción de criterios objetivos comunes para la adopción de decisiones en materia de Recursos H umanos viene siendo una tónica habitual en los últimos tiempos, y quizás es una de las principales notas definitorias de la política de Recursos H umanos. Por su parte, la introducción de sistemas de evaluación del rendimiento y su incentivación mediante la consecución de objetivos es uno de los aspectos que conforman dicha política y, por ello, consideramos conveniente profundizar en este punto.

\section{La incentivación del} rendimiento, eje de la política
de recursos humanos de la
Jefatura Central de Tráfico

Como decíamos al principio de este artículo, la política ge neral de Recursos $\mathrm{H}$ umanos que viene siguiéndose en el 0 rganismo Autónomo Jefatura Central de Tráfico se basa, entre otras premisas, en la racionalización y la objetivación, para un mejor aprovechamiento de los recursos, utilizando para ello las estrategias y mejores prácticas necesarias que permitan la optimización tanto de la calidad en el servicio al ciudadano, como de las condiciones de trabajo de los empleados públicos que en él prestan sus servicios.

La Jefatura Central de Tráfico es consciente de su función de proveer a los ciudadanos de servicios.

«Los ciudadanos, por su parte, cada vez demandan más servicios y, al propio tiempo, exigen que sus aportaciones, ya sean directas o tributarias, se utilicen eficientemente. Los ciudadanos tienen, por tanto, derecho a recibir las prestaciones y servicios públicos con niveles de calidad adecuados a sus expectativas, de rivadas de su doble condición de usuarios y contribuyentes».

Ésta es la reflexión que realiza Evencio G ON ZÁLEZ DE DIOS1, de la cual comulga plenamente nuestra organización. El mismo autor, alude a que, como ya señalaba la OCDE en 1987, se trata de poner la «Administración al servicio del público», puesto que el ciudadano espera de la Administración Pública que responda a sus necesidades. Por ello, dice, «en el ámbito de la U nión Europea y de la OCDE, las Administraciones nacionales vienen incluyendo en sus procesos de reforma de la "gestión de calidad" como una opción para avanzar en la mejora de la gestión pública y satisfacer las demandas ciudadanas. La "gestión de calidad" está orientada a los usuarios, a la obtención de resultados acordes con lo que ellos esperan, y se basa en la mejora continua de la prestación del servicio, a partir del análisis del cumplimiento de objetivos y la evaluación de los resultados obtenidos y de la satisfacción de los propios usuarioss.

El M inisterio de Administraciones Públicas, dentro de sus actuaciones encaminadas a promover la mejora continua de los servicios públicos, y con el fin de desarrollar los contenidos de la LOFAGE, ha planteado un Plan de Calidad, cuyos aspectos básicos se regulan en el Real $D$ ecreto, que regula las $C$ artas de Servicios y establece los Premios a la Calidad y a las M ejores Prácticas, publicado en el Boletín O ficial del Estado de 10 de agosto de 1999. Precisamente, resulta clarificador que junto a la regulación de las Cartas de Servicios y la C reación de los Premios a la Cali- 
dad y a las M ejores Prácticas, la promoción de la autoevaluación de unidades administrativas sea uno de los tres pilares en los que se cimenta el Plan deCalidad.

Siguiendo a LöFFLER, la Jefatura Central de Tráfico aborda - junto al resto de estrategias que componen su política de Re cursos Humanos- la evaluación del rendimiento dentro de la denominada Nueva Gestión Pública, como marco de investigación e iniciativas para la renovación de la organización y la mejora del servicio público encomendado, dentro de un entorno cultural caracterizado, de una parte, por la aspiración de sus gestores a los valores de autonomía, responsabilidad y evaluación de resultadosy, de otra, por el marco de referencia de la Calidad.

Una política de incentivación del rendimiento necesita un contexto apropiado, con plantillas dimensionadas, equipos de trabajo proporcionados, puestos de trabajo con cuotas retributivas competitivas con las del resto de la Administración del Estado, con polivalencia funcional entre distintos puestos de trabajo, etc... Por ello, se consideró que los programas de evaluación del rendimiento necesitaban previamente y como presupuesto de base el planteamiento de otras medidas previas ante las instancias competentes.

Como decimos, analizada la situación existente, la Jefatura Central de Tráfico necesitaba la puesta en práctica de varias medidas urgentes en materia de recursos humanos, con la finalidad de prestar un servicio al ciudadano con la eficacia y eficiencia propias de una administración moderna. Se trata de líneas estratégicas de actuación que en su conjunto componen el denominado Plan Integral de Recursos H umanos, que fue consensuado y negociado con la representación sindical nacional acreditada en la M esa del Plan Integral de Recursos Humanos de la Dirección General de Tráfico, con la firma de cinco de las seis organizaciones sindicales presentes en la misma. El contenido de las propuestas sirvió de base para el documento remitido el 23 de noviembre de 2001 a la Dirección General de la Función Pública.

\section{El cálculo de cargas de trabajo}

La Jefatura Central de Tráfico dispone de unos sistemas y programas informáticos que permiten la contabilización de los principales trámites que se realizan en las J efaturas Provinciales y Locales de Tráfico, de modo que se conocen las cargas de trabajo de cada U nidad y la que corresponde a cada empleado público que presta servicio en la misma. Este sistema de medición basado en criterios objetivos viene siendo utilizado para la toma de decisiones en materia de Recursos H umanos (provisión, desconcentración y creación de puestos de trabajo, contratación de personal laboral y de personal interino, asignación del presupuesto

\begin{tabular}{|c|c|}
\hline Tipo de trámite & Valor ponderado \\
\hline M atriculación de vehículos................................. & 1,8 \\
\hline Transferencia de la titularidad de un vehículo....... & 1,8 \\
\hline $\begin{array}{l}\text { Declaración de aptos y no aptos en pruebas de } \\
\text { aptitud para la obtención de autorizaciones }\end{array}$ & \\
\hline 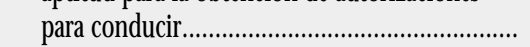 & 2,9 \\
\hline Bajas de vehículos. & 1,0 \\
\hline Revisiones de permisos y licencias de conducir ..... & 1,0 \\
\hline Duplicados de permisos de conducir y de & \\
\hline 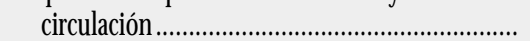 & 1,0 \\
\hline 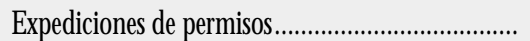 & 1,0 \\
\hline 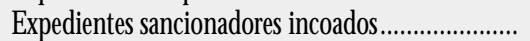 & 1,0 \\
\hline 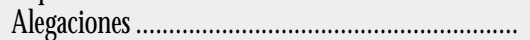 & 1,6 \\
\hline Resolución de recursos ....................................... & 2,2 \\
\hline Suspensiones de permisos y licencias de conducir ... & 2,9 \\
\hline
\end{tabular}

provincial de incentivos al rendimiento paras las l efaturas en las que no está implantado aún el sistema CAAP y el CEX, cuantificación del número de dotaciones de cobro que corresponde a cada J efatura, etc...).

Para ello, se toman en cuenta los trámites más representativos, se ponderan en función de su complejidad y se dividen entre las plantillas, no contabilizándose aquellos puestos que, por la peculiaridad de sus funciones, se considera no tienen una incidencia directa en la realización de cada trámite. En el Cuadro anterior se indican los tipos de trámites seleccionados para ser tenidos en cuenta y la ponderación realizada por los Jefes Provinciales coordinadores y la Unidad de Inspección y Calidad de los Servicios de la Secretaría G eneral.

Analizando el volumen de trámites y las plantillas, podría concluirse que:

1. Existiría una carga de trabajo por funcionario denominada «carga 1», que sería el resultado de dividir el volumen total de trámites de un año entre la plantilla teórica de la Unidad, exceptuando los puestos anteriormente indicados.

2. Existiría una carga de trabajo por funcionario denominada «carga 2», que sería la correspondiente al colectivo del área administrativa y de atención al público (CAAP), y que se obtendría de dividir el volumen total de trámites de un año pero con aptos y no aptos de pruebas de aptitud para la obtención de autorizaciones administrativas para conducir ponderados a valor 1 (no 2,9, como en el caso anterior, por entender que dicho valor 1 es el que corresponde al trabajo de anotación de presentación a examen y mecanización del resultado del mismo por el personal de Jefatura por cada apto o no apto realizado). El otro 1,8 restante hasta alcanzar el 2,9 es el que correspondería a la realización y calificación de la prueba de aptitud, llevada a cabo por el colectivo del área de prue- 


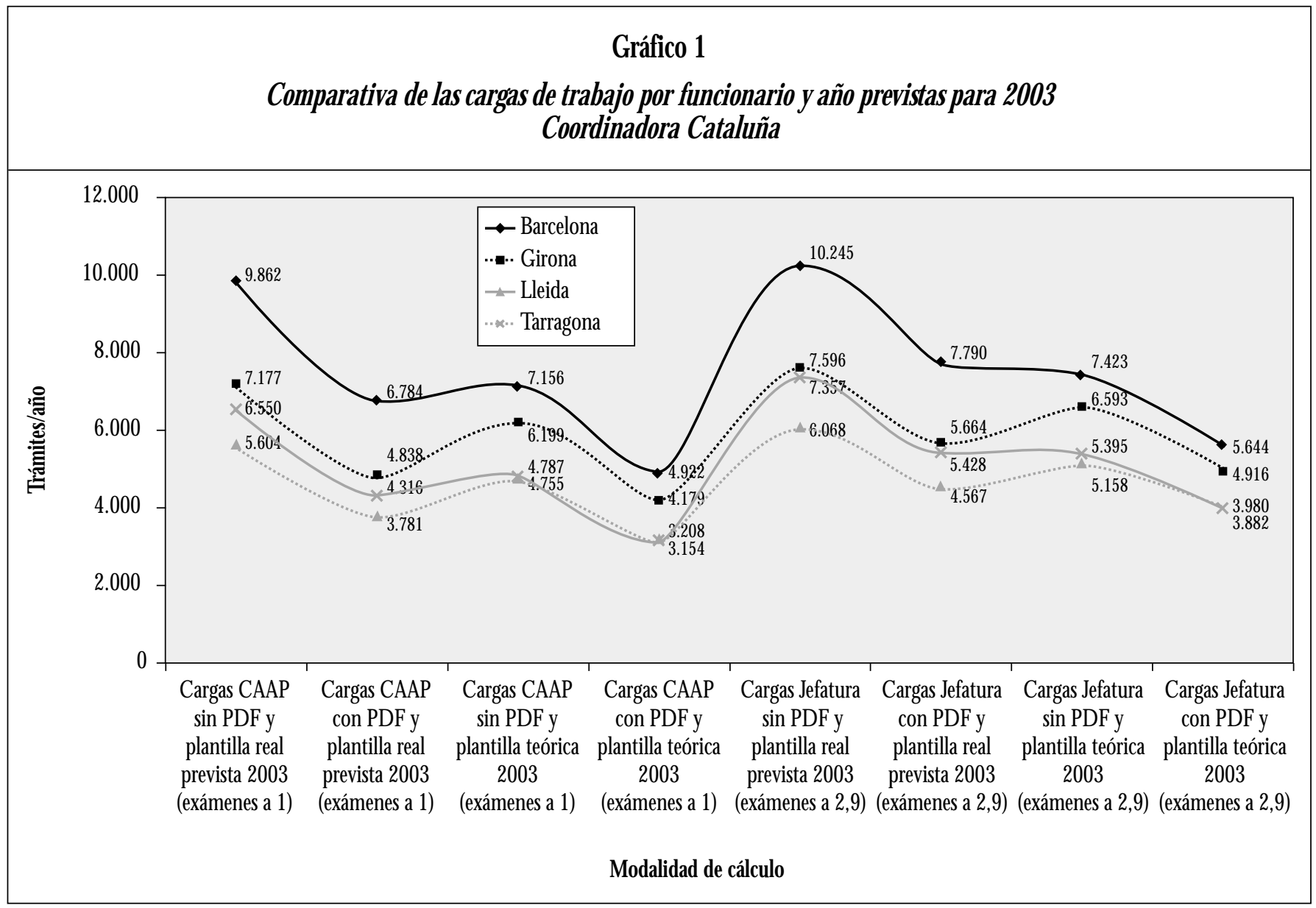

bas de aptitud para la obtención de autorizaciones administrativas para conducir (CEX). Para el cálculo de esta «carga 2» se excluiría por tanto al personal examinador y examinador-coordinador (integrante del CEX).

La «carga 1» es la que se tiene en cuenta para efectuar la asignación anual del presupuesto de incentivos al rendimiento en las Jefaturas donde todavía no está implantado el sistema de incentivos CAAP y CEX, de acuerdo con lo que más adelante veremos. Como lo que se pretende con esta fase preliminar es dimensionar las plantillas teóricas en el sentido de adecuar los medios humanos a la demanda ciudadana, vamos a tomar como base la «carga 2». Esta «carga 2» es una carga máxima teórica, dando por hecho que existen circunstancias que hacen que la carga real sea superior (es el caso de las vacantes, del índice de jornadas perdidas, etc,...). Por ello, a esta «carga 2» la llamaremos «carga de trabajo máxima por funcionario» (en ade lante, CM F).

El proceso se realiza mediante una compleja hoja de cálculo y tiene en cuenta las siguientes premisas:
a) Secalculan las CM F.

b) A través de los estudios realizados por las Jefaturas provinciales coordinadoras y la Unidad de Inspección y Calidad de los Servicios se ha determinado que la CM F en ningún caso debería ser superior a 6.000 trámites/año, si se pretende prestar un servicio de calidad.

c) Se cruzan las CM F comparándolas con las máximas de 6.000 trámites/año.

d) Secalcula el incremento necesario en el número de puestos para que la CM F no sea superior a 6.000 trámites.

e) Se calculan los puestos de trabajo de jefe de negociado y de jefe de sección, necesarios para mantener una adecuada estructura de los equipos de cada U nidad.

En su momento se arbitraron hasta ocho sistemas distintos de cálculo de cargas de trabajo, dependiendo de si se tomaban en cuenta las plantillas teóricas o las reales, el trabajo completo de la Unidad englobando la tramitación administrativa y el área de pruebas de aptitud o por separado, y el modo de influir las tecnologías de la información en la realización de los procesos productivos. La posición que ocupa una J efatura con respecto a las demás varía en función del sistema de cálculo elegido. En el gráfico 1 se observa lo que ocurriría, por ejemplo, 


\section{Gráfico 2}

\section{Previsiones de cargas de trabajo del colectivo CAAP (modelo 4) para 2003}

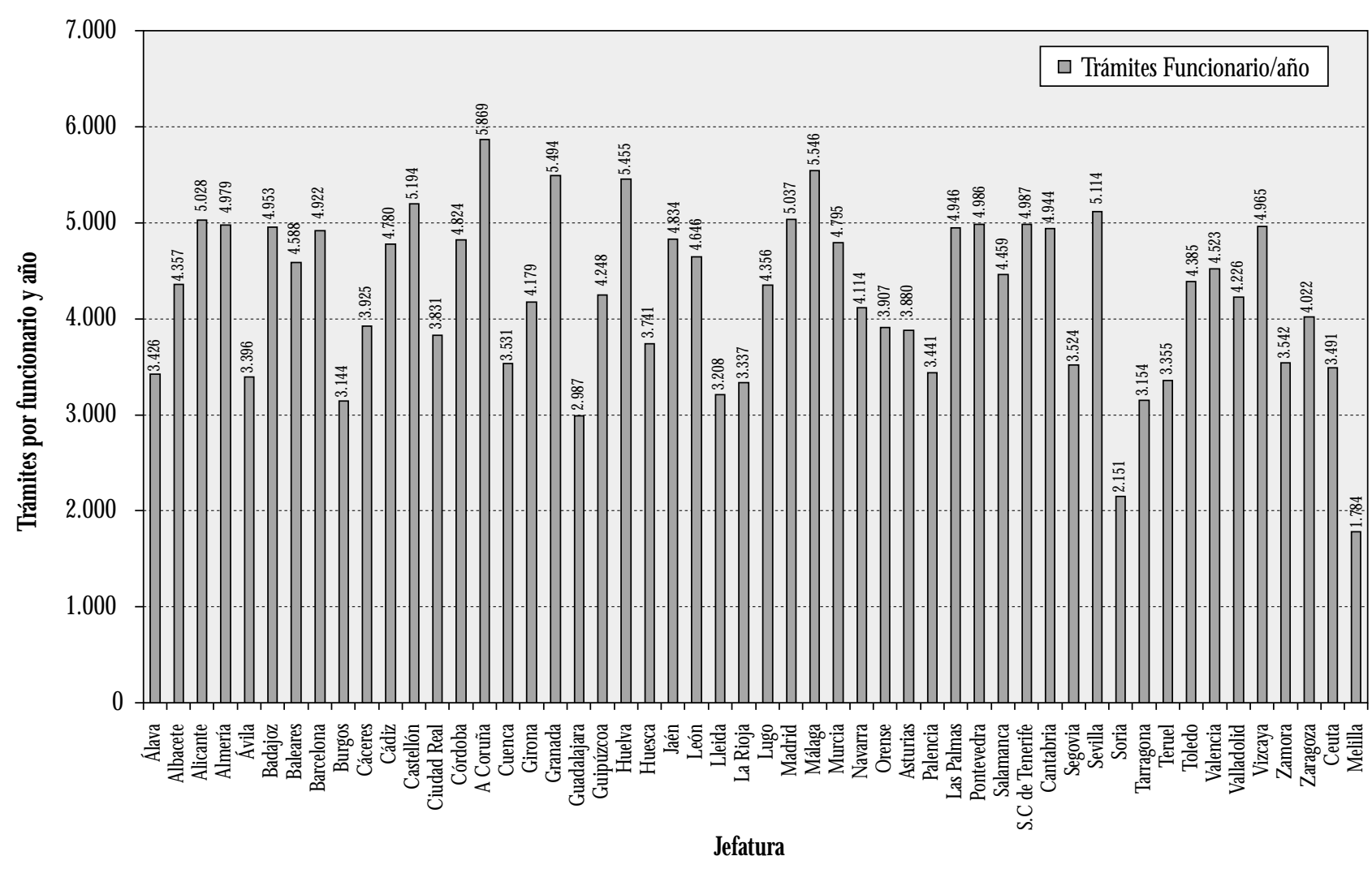

con las J efaturas Provinciales de Tráfico de C ataluña, para el año 2003.

Este último aspecto está cobrando cada vez más importancia. Con la implantación de nuevos sistemas de organización del trabajo y tecnologías de la información - que a su vez reportan nuevos parámetros e indicadores- sin duda se ha influido en la complejidad y los tiempos de realización de los trámites, reduciéndolos, alterando la situación de las cargas de trabajo de las diferentes unidades de la organización periférica.

Se ha firmado un convenio entre la Dirección General de Tráfico y el Consejo $G$ eneral de Colegios de Gestores Admini strativos, en virtud del cual las Jefaturas han etablecido protocolos deadhesión al Convenio con los Colegios respectivos.

Para simplificar la anotación informática de los datos relativosa trámites de vehículos y consulta de antecedentes, los gestores administrativos proporcionan junto con toda la documentación exigida, una hoja en la que constan los datos quedeben ser anotados, codificados con un código de barras bidimensional (man- cha PDF), de acuerdo con el formato de registro definido por la Dirección G eneral deTráfico.

Los colegios de gestores administrativos recogen los expedientes de sus col egiados, generan la hoja con la mancha PDF y entregan la documentación en la Jefatura. En las Jefaturas, con lectores de código PD F, verifican la documentación y completan la transacción leyendo los datos necesarios directamente de la mancha PDF.

Este sistema se está utilizando para matriculaciones, bajas, transferenciasy obtención de informes de vehícul os.

Conforme al criterio emanado de la Unidad de Inspección y Calidad de los Servicios: 6 matriculaciones con PD F equivalen a 1 sin PDF; 5 transferencias con PDF equivalen a 1 sin PDF; y 8 bajas con PDF equivalen a 1 sin PDF.

Por ello, a la hora de calcular la carga de trabajo para determinar las cuantías de productividad a percibir por cada efectivo CAAP en las Jefaturas Provinciales y Locales de Tráfico se ha considerado conveniente dividir los trámites totales (distinguiendo los hechos con y sin PDF), entre la plantilla teórica pre- 
vista para 2002, pero con los aptos y no aptos de pruebas para la obtención de autorizaciones administrativas para conducir sin ponderar, esto es, con valor 1, por entender que es el trabajo que corresponde realizar al personal del área administrativa (el resto hasta 2,9, se utilizaría para calcular las cargas del área de pruebas para la obtención de autorizaciones administrativas para conducir). Ésta sería la carga de trabajo del colectivo CAAP, es decir, la que se correspondería con el modelo número cuatro de los ocho indicados en el gráfico anterior. Por tanto, no se ha optado por tomar en cuenta las plantillas reales, puesto que ello supondría asumir que el CAAP y el CEX conllevarían un contexto de absentismo cuando, al contrario, estos sistemas tienen como uno de sus objetivos fomentar la asistencia a J efatura a través de la incentivación económica.

Con este sistema de cálculo de cargas - y por tanto, de de terminación del importe diario CAAP, como veremos más ade lante- de acuerdo con el model o 4, la comparativa interprovincial quedaría conforme indica el gráfico 2 ( en el que puede apreciarse que, en la actualidad no existirían Jefaturas con cargas superiores a los 6.000 trámites ponderados por funcionario y año con plantilla teórica, habiendo culminado con éxito el proceso de dimensionamiento de plantillas.

Los diferentes sistemas de cálculo de cargas de trabajo de cada Unidad y de los empleados públicos que la integran, permite arbitrar soluciones ajustadas a cada tipo de problema: para dimensionar plantillas teóricas de la U nidad o de un colectivo de la misma, para asignar el presupuesto de productividad en caso de no tener implantado el CAAP y el CEX, para desconcentrar puestos de trabajo o reforzar con efectivos en comisión de servicios, etc.

\section{El paso del sistema tradicional a la evaluación del rendimiento por criterios objetivos en la organización periferica: el CAAP y el CEX}

\section{La asignación anual}

En una primera fase (1998) se puso en práctica un primer siste ma para la asignación del presupuesto anual de productividad sobre la base de criterios objetivos. Para ello, se calculó la carga de tra bajo por funcionario y año, y se introdujeron condicionales y coeficientes correctores para limitar variaciones drásticas en la asignación con respecto a la del año anterior (previo a la implantación de este nuevo sistema). En la segunda fase (1999), para calcular el presupuesto de cada Jefatura para 1999, de acuerdo con el sistema de cálculo de cargas tradicional, se aplicó al presupuesto de 1998 la misma variación experimentada en la carga de trabajo con respecto a 1997, entendiendo que para el año 1999 la tendencia sería la misma. En la actualidad, coexisten dos tipos de Jefaturas: las que tienen implantados los sistemas CAAP y CEX y las que no. En este segundo caso, la asignación del presupuesto se realiza conforme al sistema tradicional, si bien se ha superado el concepto de «absentismo», y se toma en cuenta el denominado «índice de jornadas perdidas», mucho más completo, por cuanto contempla cualquier aspecto que incide en la cobertura de los puestos y la presencia del personal en Jefatura (absentismo, vacantes, comisiones de servicio, etc...). D e este modo se calcula la diferencia entre la plantilla «teórica» y la plantilla «real».

Las divergencias entre la asignación prevista y las necesidades reales de las J efaturas Provinciales en productividad, derivadas de situaciones imprevistas, se solucionan mediante asignaciones adicionales no consolidables para las que se analizan las causas al egadas por los titulares de las U nidades, siempre sobre la base de dar un mejor servicio al ciudadano.

\section{La productividad por rendimiento en áreas estratégicas! contenido}

D esde el 1 de febrero de 2002 se viene siguiendo una experiencia en las Jefaturas Provinciales de Barcelona, Sevilla y Cantabria, y en la Local de Sabadell, consistente en una productividad por rendimiento en el área administrativa y de atención al público (CAAP) y en el área de pruebas de aptitud - CEX - en todas menos en Sevilla). Los criterios sobre los que se viene asentando son:

\section{EICAAP}

\section{A) Jornada ordinaria}

El objetivo es la mejora en la calidad del servicio al ciudadano, mediante la consecución del «retraso cero» en el área administrativa y de atención al público, con el compromiso por parte de los empleados públicos de absorber la demanda ciudadana en jornada ordinaria. A cada Jefatura se le asigna un presupuesto diario equivalente a multiplicar el número total de puestos del área CAAP (cubiertos o no), por un importe diario en función de la carga de trabajo que corresponda a los emple- 
ados públicos de esa Jefatura. Si la U nidad consigue el «retraso cero», cada empleado público que asista ese día percibe el importe diario. El presupuesto no gastado ese día por los puestos vacantes y las ausencias genera una bolsa que se puede repartir entre los presentes.

El reparto se está real izando de formas diversas en los diferentes equipos de trabajo: a) una cantidad igual para todos los asistentes; b) una cantidad igual entre quienes han colaborado directamente en la consecución de los objetivos fijados; c) sólo a quienes superen la media de trámites realizada por el equipo ese día; d) sólo a quienes además no incumplan la jornada laboral (retrasos) y a quienes cumplimenten la ficha diaria de tareas desempeñadas.

Los puestos que no sean CAAP podrán percibir esta productividad en la mismas condiciones que el resto del equipo siempre que el Jefe de Unidad disponga de la citada bolsa. Es decir, el Jefe de Unidad puede optar bien por repartir, bien por destinar la bolsa a empleados públicos no CAAP, bien ambas cosas, siempre con el límite de presupuesto diario.

El importe diario no se percibe en casos de ausencia salvo causas expresamente tasadas y consensuadas con la representación sindical.

\section{B) Apertura al público por la tarde}

El objetivo es el mismo que para el caso del CAAP mañana, pero con el compromiso adicional de formar equipo en jornada de tarde durante un año. D e este modo, el colectivo CAAP percibirá un importe de productividad por la realización de su trabajo en jornada de mañana, y otro por cada tarde de trabajo, existiendo dos tipos de jornadas, en función del día de la semana: jornada general, o jornada partida. Los puestos de trabajo que por la especificidad de sus funciones (por ejemplo, cajero) perciban un complemento de productividad en jornada de mañana, percibirán la parte proporcional por cada tarde de trabajo.

Las unidades en que se deba atender al público por la tarde, el número de tardes a la semana y la distribución del horario partido se establece previo análisis de la U nidad de Inspección y Calidad de los Servicios y la Subdirección General de Sistemas deInformación y O rganización de Procedimientos.

\section{EL CEX}

El objetivo también es la mejora en la calidad del servicio al ciudadano, mediante la consecución del «retraso cero» en el área de pruebas de aptitud, en este caso con el compromiso por parte de los empleados públicos de la absorción de la demanda de pruebas de aptitud en jornada ordinaria. Se encomiendan - por cada efectivo CEX - 16 pruebas prácticas ponderadas en función de su duración y complejidad (de manera que una prueba para obtener un permiso profesional equivale a 1,8 pruebas ordinarias), se fija un valor / prueba y se percibe un importe equivalente a multiplicar dicho valor por cada prueba realizada. Cuando un integrante del equipo no asiste al trabajo, sus pruebas se reparten entre el resto de asistentes, en cuyo caso el valor que se abona por cada prueba que supere las dieciséis es prácticamente el doble. En cualquier caso, el número de pruebas a realizar nunca será superior a 20.

Al igual que en el CAAP, la percepción está condicionada a la asistencia al trabajo, y están tasadas las mismas causas que hacen considerar una ausencia como justificada.

La implantación del sistema se realiza dentro de un contexto de plantillas dimensionadas, de modo que la capacidad de examen en jornada ordinaria sea la necesaria para satisfacer la demanda ciudadana de este servicio. Los efectivos CEX que realicen pruebas de conocimientos (exámenes teóricos) o realicen inspecciones de centros colaboradores de Tráfico, percibirán la misma cantidad que quienes realicen pruebas de circulación. En casos de menor demanda ciudadana, los efectivos CEX se destinan a labores CAAP percibiendo dicha productividad.

\section{Losincentivosa la asistencia para el CAAP y el CEX}

Las Unidades disponen de un presupuesto trimestral equivalente a multiplicar por $18 €$ el número de puestos del colectivo. Se concede hasta $153,25 €$ a quienes no se ausenten al trimestre más de 4 horas 40 minutos en caso de jornada ordinaria, 0 de 5 horas para mayor jornada. Caso de que el número de efectivos con derecho al incentivo sea superior al que permitiría el presupuesto, el importe se distribuirá a prorrata entre todos ellos. Si por el contrario quedara presupuesto sin adjudicar, se acumula para el trimestre siguiente. Se consideran ausencias justificadas las mismas que para el caso de percepción del importe diario de productividad, añadiendo las vacaciones anuales y días de permiso por asuntos particulares.

\section{El premio anual al mayor rendimiento}

Está prevista la concesión de $60 €$ por cada efectivo a la Unidad con mayor rendimiento por efectivo CAAP y año. 


\section{Gráfico 3}

Tiempo medio de espera ciudadana en jornada de mañana en la Jefatura Provincial de Tráfico de Barcelona

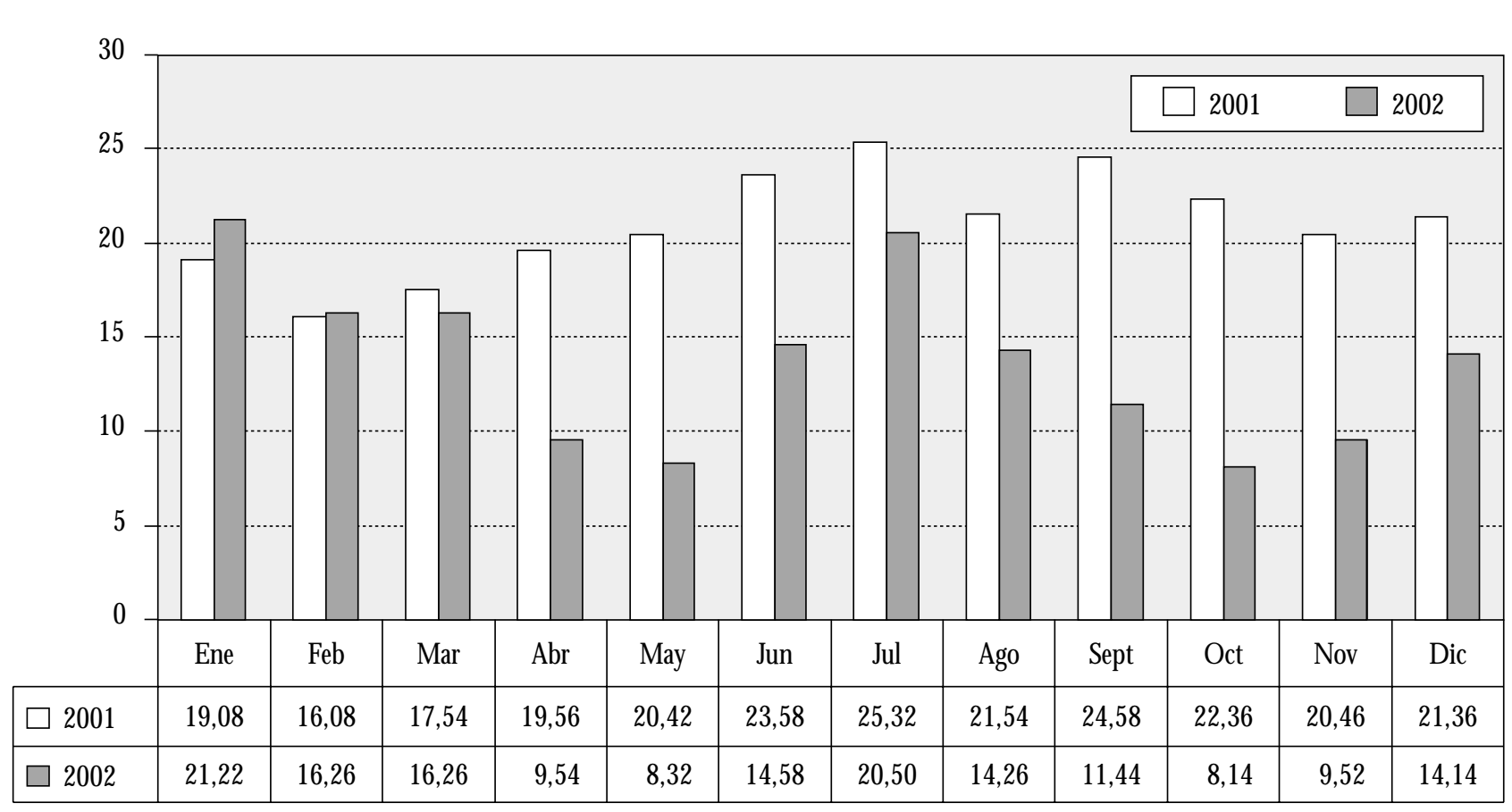

\section{Gráfico 4}

Porcentaje de ciudadanos, atendidos en menos de diez minutos en la Jefatura Provincial de Tráfico de Barcelona

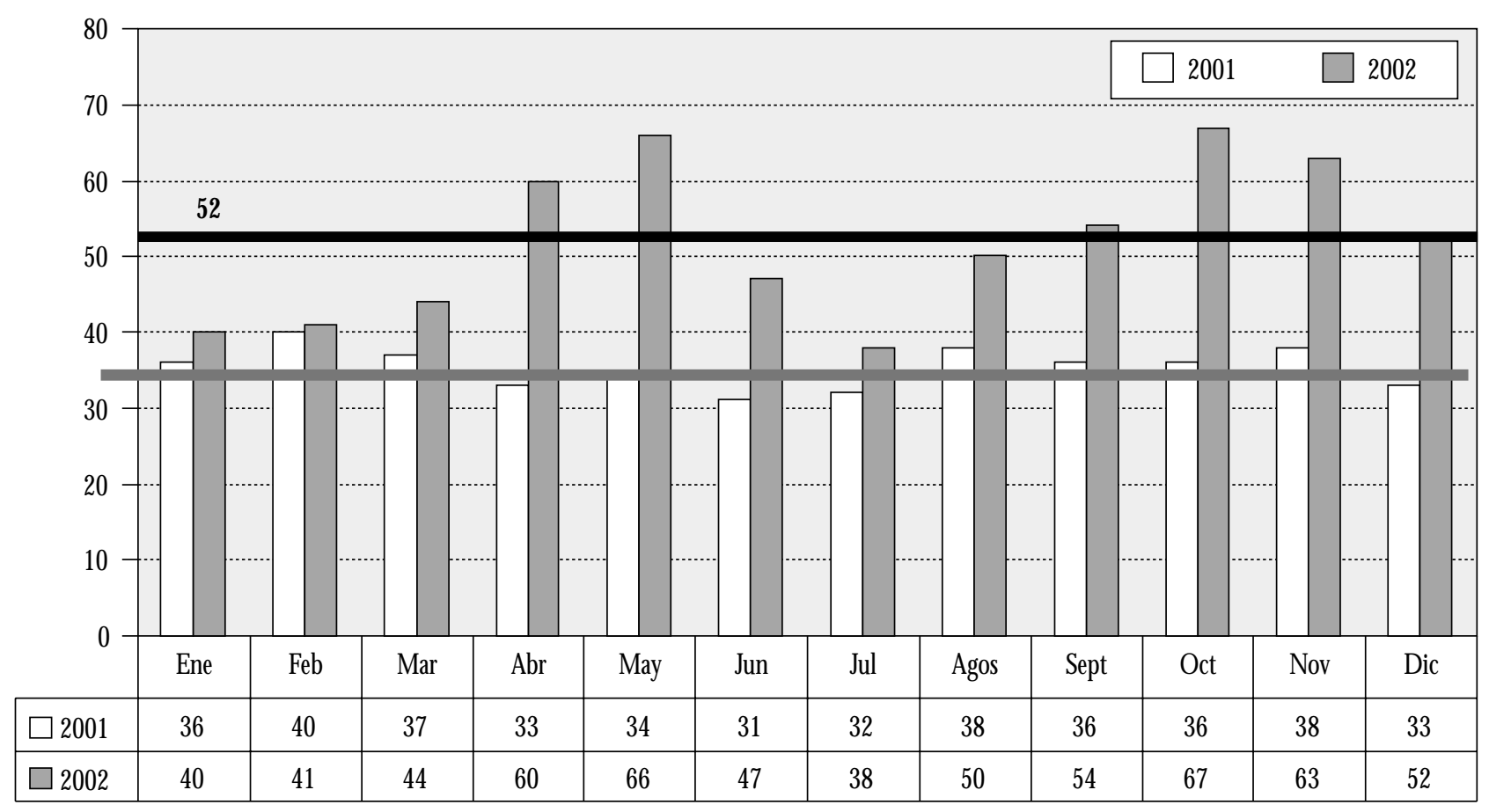




\section{¿Q ué ha supuesto la implantación de estos sistemas?}

EI CAAP está implantado en las J efaturas Provinciales de Tráfico de Barcelona, Sevilla, Cantabria y Sabadell desde el 1 de febrero de 2002. EI CEX está implantado desde la misma fecha en todas las Unidades citadas excepto en Sevilla, donde aún no está adecuadamente dimensionada la plantilla a la demanda ciudadana. La apertura al público por la tarde es, actualmente, de tres tardes (martes, miércoles y jueves) en Barcelona, y una tarde (los jueves) en Sevilla, Cantabria y Sabadell.

\section{Reduce el tiempo medio de espera de los ciudadanos}

Si tomamos como ejemplo la Jefatura de Barcelona, nos encontramos con que la implantación del CAAP se realizó en un contexto de incremento de la demanda ciudadana en el 7,84\% respecto al año 2001 (de 442.752 hemos pasado a 477.467 ciudadanos atendidos). Aunque todo auguraba un incremento en el tiempo medio de espera de los ciudadanos en jornada de mañana, la implantación del CAAP ha permitido reducirlo un $47,79 \%$, pasando de 25,15 a 13,13 minutos. Podemos verlo más claramente en el siguiente gráfico 3.

Además, pese a ese inquietante escenario de incremento de la demanda ciudadana, se ha conseguido aumentar el porcentaje de ciudadanos atendidos en menos de diez minutos, que ha pasado de ser del 34 al 52\% (gráfico 4).

Este aumento ha sido aún más espectacular en el caso de Caja, donde el 70,41\% de los ciudadanos han sido atendidos en menos de diez minutos (cuando este porcentaje era del 38,3\% en el año 2001).

\begin{tabular}{|cccc|}
\hline Años & $\begin{array}{c}\text { Total clientes } \\
\text { atendidos }\end{array}$ & $\begin{array}{c}\text { Tiempo medio } \\
\text { de espera }\end{array}$ & $\begin{array}{c}\% \text { atendidos en } \\
\text { menos de 10 } \\
\text { minutos }\end{array}$ \\
\hline $2001 \ldots \ldots \ldots \ldots \ldots . . .$. & 224.882 & $9^{\prime} 40^{\prime \prime}$ & 38,30 \\
$2002 \ldots \ldots \ldots \ldots \ldots . . .$. & 245.116 & $8^{\prime} 50^{\prime \prime}$ & 70,41 \\
Variación ............... & $+10 \%$ & $-8,6 \%$ & $+83,81 \%$ \\
\hline
\end{tabular}

\section{Permite el establecimiento de objetivos (retos) alcanzables por la organización}

Las Jefaturas piloto se habían fijado los objetivos a cumplir, y el plazo máximo en que se deben conseguir. Para ello, por ejemplo, la Jefatura Provincial de Tráfico de C antabria, con la implantación del nuevo sistema de productividad para toda el área administrativa y de atención al público, además de la fijación de objetivos y los correspondientes indicadores, ha realizado mejoras organizativas: unificación de las ventanillas de vehículos y conductores, apertura de un puesto de atención al público adicional, realización de un control diario de la resolución de los expedientes de particulares y gestores administrativos y un control mensual dela realización de otros trámites.

La fijación de objetivos para cada unidad de gestión y el compromiso de los empleados públicos son elementos esenciales para que el 0 rganismo consiga los objetivos que asuma en la carta de servicios.

\section{Permite conseguir el denominado «retraso cero».}

Las Jefaturas han conseguido cumplir los objetivos fijados pese a que en algunos casos se han experimentado incrementos en el volumen de cierto tipo de trámites, de hasta un $40 \%$.

\section{EI CAAP de tarde permite satisfacer la demanda de los ciudadanos acercándoles más las oficinas administrativas}

Sorprende ver cómo se va incrementando el número medio de ciudadanos atendidos por la tarde, por ejemplo en la jefatura de Barcelona, lo cual da una idea de la aceptación de esta medida.

\section{Mejora la valoración de los ciudadanos sobre la calidad del servicio}

La valoración realizada por los usuarios de la Jefatura Provincial de Tráfico de Barcelona, respecto al servicio recibido, ha sido la siguiente:

\begin{tabular}{|ccccccccc|}
\hline Año 2002 & Feb & Mar & Abr & May & Jun & Sep & Oct \\
\hline Calidad de la información ......... & 7,7 & 8,2 & 8,5 & 8,3 & 8,6 & 8,5 & 8,5 \\
Calidad dela atención ……..... & 7,9 & 8,8 & 9,1 & 8,8 & 9,0 & 9,0 & 9,0 \\
Tiempo de espera ................. & 6,7 & 6,5 & 7,4 & 7,8 & 7,7 & 7,6 & 7,6 \\
\hline
\end{tabular}



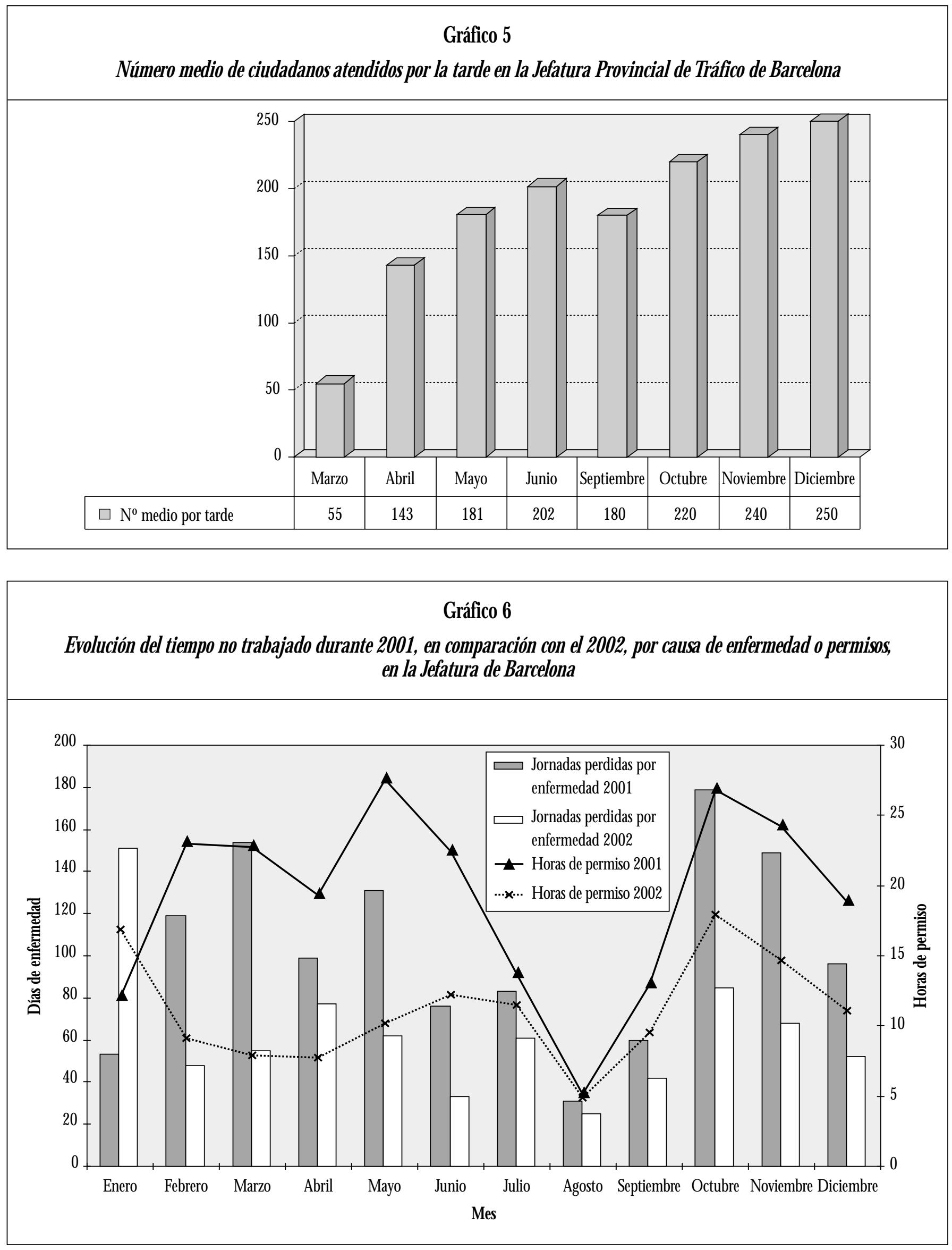


\section{Gráfico 7}

\section{Valoración del CAAP por los funcionarios de la Jefatura Provincial deTráfico de Sevilla}

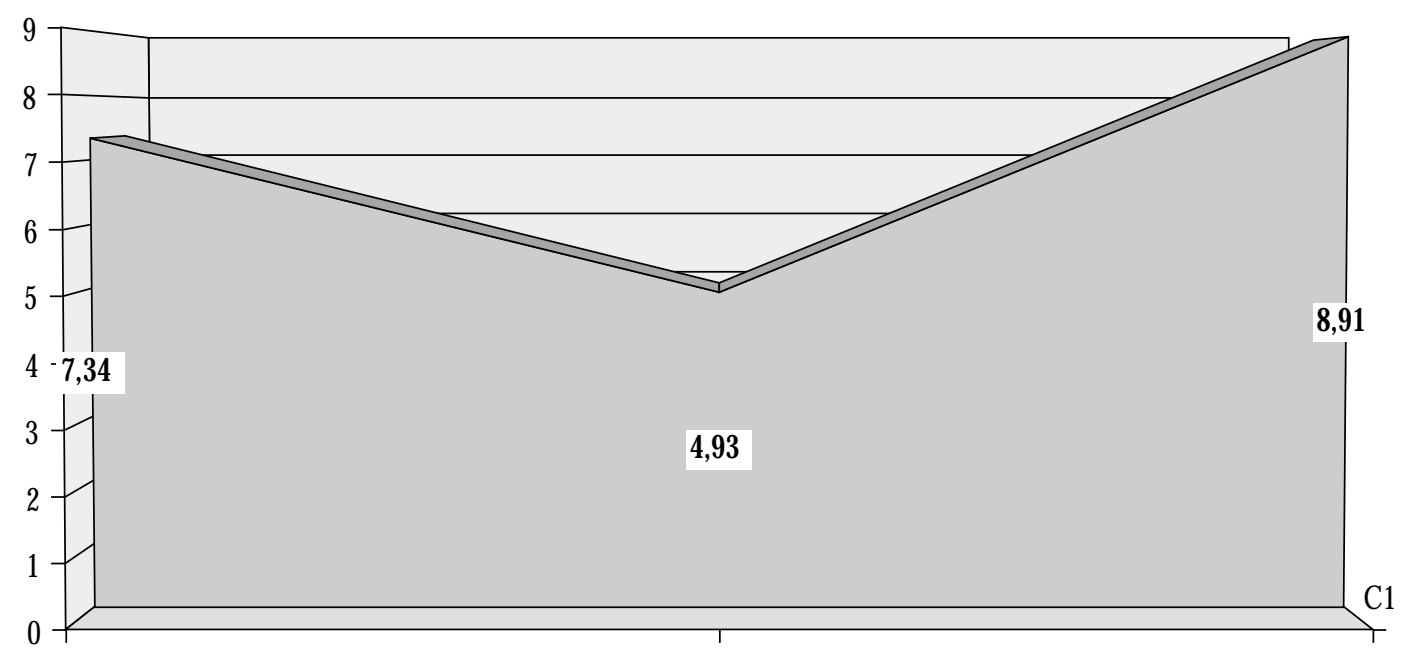

\section{Se reduce notablemente el número de jornadas perdidas}

\begin{tabular}{|lrrr|}
\hline & $\mathbf{2 0 0 1}$ & $\mathbf{2 0 0 2}$ & Variación \\
\hline Enfermedad .......... & 1.177 & 608 & $-49,4 \%$ \\
Permisos .............. & 742 & 400 & $-46,1 \%$ \\
Total .................. & 1.919 & 1.108 & $-47,5 \%$ \\
\hline
\end{tabular}

El cuadro anterior refleja el porcentaje de variación en las jornadas perdidas por enfermedades sin baja y permisos durante los años 2001 y 2002 en Barcelona, alcanzando hasta un 47,5\% de reducción, lo cual influye notablemente en la capacidad de respuesta a la demanda ciudadana. Este dato, aplastante de por sí, hay que encuadrarlo en un escenario que se mostraba claramente desfavorable con respecto al año 2001, ya que en enero de 2002 el índice dejornadas perdidas fue notablemente superior al de enero de 2001. En el gráfico 6 observamos la comparativa de ambos años de las jornadas perdidas por enfermedad y del tiempo dedicado a permisos, comprobando claramente el impacto positivo de las productividades CAAP y CEX.

La productividad CAAP y CEX se percibe en función de la asistencia o no al trabajo, lo que ha llevado a una reducción notable del número de jornadas perdidas en las J efaturas piloto. La asistencia de una mayor proporción de los efectivos de cada unidad, produce una mejor distribución de la carga de trabajo permite conse guir los objetivos de calidad fijados para la unidad.

\section{Mejora el clima laboral}

El diseño del sistema de productividad CAAP y CEX fuedebatido con la representación sindical acreditada en el 0 rganismo, y cuenta con el apoyo y la firma de cinco de los seis sindicatos presentes (CC.00, UGT, CSI-CSIF, CIG y ELA). La prueba ha sido en general muy bien recibida por todo el equipo CAAP y CEX. Los representantes de las centrales sindicales también la han valorado favorablemente y apuestan también por su extensión, aunque insisten en reconsiderar el, a su juicio, rigurosísimo concepto de absentismo.

Como vemos en el gráfico 7, en la Jefatura de Sevilla se realizó un sondeo entre el personal CAAP, acerca del propio sistema CAAP, solicitando opinión sobre tres asuntos clave. Los datos son contundentes: el personal CAAP valora muy positivamente el sistema, y apuesta por su extensión al resto de Jefaturas donde no esté aún implantado. No obstante, solo se ha dado un aprobado en el apartado «motivación», debido a que gustaría que las cuantías fueran superiores a las establecidas.

\section{Reduce el tiempo de exposición a riesgos laborales y atenúa los siete factores de riesgo psicosocial (INSHT)}

La no necesaria vinculación de una mayor retribución con una prolongación de la jornada de trabajo y la incentivación del rendimiento, hace que el personal pueda cumplir sus objetivos dedican- 
do más tiempo a la familia y ocio, lo cual contribuye a afrontar las responsabilidades laborales en mejores condiciones psicofísicas.

\section{Perspectivas de futuro}

Por supuesto, el O rganismo apuesta por la continuidad de los sistemas CAAP y CEX en las J efaturas en que ya está implantado, y por su extensión al resto, siempre en función de las disponibilidades presupuestarias y de la financiación adicional del presupuesto de productividad que se consiga de los M inisterios correspondientes. Además, se prevé analizar otras dos áreas estratégicas: la educación vial y la investigación de la seguridad vial, con lo cual la práctica totalidad de las plantillas de la organización periférica estará incluida en sistemas de evaluación del rendimiento por criterios objetivos.

En cuanto al cál culo de cargas de trabajo, se está ampliando a 36 el análisis de la evolución de los 11 trámites tipo tenidos en consideración, comparándolos desde 1997, con lo que se va a disponer de una visión cada vez más completa.

Existe una notable coincidencia entre el Acuerdo Administración-Sindicatos para la modernización y mejora de la Administración Pública en los años 2003-2004 y las líneas generales de la política de Recursos H umanos de la Jefatura Central de Tráfico, lo que anima a continuar con nuestra línea de actuación. No obstante, el M inisterio de Administraciones Públicas ha apostado decididamente porque la apertura al ciudadano sea ininterrumpida e incentivada no a través de los créditos de productividad, sino de la elevación de los complementos específicos de los puestos de trabajo que conformen los equipos necesarios. N uestro 0 rganismo se ha acogido al Acuerdo y en la actualidad se está atendiendo al público en once Jefaturas y es inminente la apertura en otras cuatro (con ello se abarcaría a las 14 J efaturas con mayor volumen de tramitación a las que se añade C antabria), el sistema CAAP tarde está siendo sustituido y de hecho sólo se aplica ya transitoriamente en Sabadell. Q uiere ello decir que el CAAP continuará aplicándose y extendiéndose en la organización periférica, pero sólo en su modalidad de mañanas, y que la atención al público en jornada de mañana y tarde se realizará no utilizando el complemento de productividad, sino con los equipos de trabajo de quienes han visto modificados sus complementos espećíficos para partir su jornada.

0 tro ejemplo de la decidida apuesta de la J efatura Central de Tráfico por reordenar los créditos de productividad para conseguir elevar el nivel de calidad del servicio público y mejorar las políticas de motivación e incentivación de los empleados públicos es que la productividad por objetivos se está implantando también en la Subdirección G eneral Adjunta de Recursos Humanos de la J efatura Central de Tráfico desde el 1 de mayo de 2003, con arreglo a un nuevo sistema que, aunque responde a la denominada «filosofía CAAP», tiene en cuenta la peculiaridad de los Servicios $C$ entrales con respecto a la organización periférica del 0 rganismo (mientras que en éstos es la atención al ciudadano la nota definitoria de la actividad, en aquellos es el empleado público el principal destinatario del servicio). Dicho sistema incentiva la participación del equipo en la consecución de los objetivos, el mayor rendimiento con respecto al estándar, la calidad del servicio, la consecución de los retos considerados estraté gicos y la presencia efectiva en el puesto de trabajo.

D e este modo, se demuestra que es posible conducir a valores unificados los correspondientes a los procesos productivos de servicios centrales y periféricos y que todos los empleados públicos tienen la oportunidad de estar incluidos en estos sistemas de incentivación sin estar limitados por el tipo de organización (central o periférica) en la que estén destinados.

Notas

* Subdirector G eneral Adjunto de Recursos H umanos de la J efatura Central deTráfico.

Bibliografía

GonzÁLEZ DE DIOS, E., «Plan de Calidad en la Administración General del Estado», en Evaluación y Calidad en las organizaciones públicas, IN AP, 2000, pp. 323-336. 
